\section{Old organs, new matrix}

\section{By Kai-Jye Lou, Staff Writer}

Research groups at Harvard Medical School and Yale University separately have developed protocols for using decellularized matrices to generate transplantable liver and lung grafts that have functional vascular systems. ${ }^{1,2}$ The findings provide further evidence that unusable donor organs could serve as the scaffold for engineering replacement organs.

The majority of scaffolds used in engineered tissue constructs are fabricated de novo from combinations of natural and synthetic biomaterials. However, such scaffolds typically lack the structural equivalent of a vascular network.

"One of the major problems in transplantation is maintaining blood flow in the organ," said Shay Soker, a professor of regenerative medicine at the Wake Forest Institute for Regenerative Medicine. "Most previous works dealt with engineered organ grafts that do not have an intact vascular network; what you get after transplanting such grafts is ischemia due to poor perfusion."

To solve the ischemia issue, the two research groups turned to decellularized lung and liver matrices as scaffolds.

Decellularization is a process in which chemicals and enzymes are used to remove the cells from an organ or piece of tissue. The resulting decellularized extracellular matrices (ECMs) are attractive scaffolds for engineering tissue constructs because they retain both the $3 \mathrm{D}$ architecture of the original part and the associated vasculature. ${ }^{3}$

Indeed, replacement bladders and tracheas built upon decellularized matrices are already being tested in patients. But these organs have relatively low functional complexity compared with other internal organs like the heart, liver and lungs. In 2008, researchers at Harvard Medical School and the University of Minnesota accomplished a challenging feat by using a decellularized heart matrix to engineer a beating rat heart. ${ }^{4}$

Now, the Harvard and Yale groups have shown that the approach is feasible with livers and lungs.

The Harvard researchers reported in Nature Medicine that they had isolated a rat liver and perfused it with an anionic detergent to create a decellularized matrix. The researchers then seeded the matrix with primary rat hepatocytes and cultured the construct in an in vitro perfusion chamber. In rats, hepatocytes within the graft remained viable and functional after being connected to the host circulatory system for eight hours.
"Our work shows that the liver itself already provides a useable scaffold for creating a replacement, so you won't have to create a scaffold from the ground up," said Korkut Uygun, corresponding author on the paper and an instructor in surgery at Harvard Medical School. "Our findings support the idea that donor organs considered unsuitable for transplantation could still provide us with a scaffold that can be used to engineer a replacement organ."

According to Uygun, the results were surprising because conventional wisdom held that the decellularization process would not work well with the liver, which has an ECM that is less sturdy than that of more solid organs like the heart and lungs.

"We had thought that the resulting graft would not have sufficient strength to handle the mechanical stresses introduced by normal blood flow," he told SciBX.

The Yale team used a different detergent solution to generate a decellularized matrix from an isolated rat lung. The researchers seeded the matrix with a mix of neonatal rat lung epithelial and microvascular endothelial cells and cultured the resulting construct in a lung bioreactor. The epithelial and endothelial cells selectively adhered to the alveolar and vasculature surfaces on the matrix, respectively.

The researchers transplanted the lung grafts into rats for up to two hours and showed that the organs carried out oxygen and carbon dioxide exchange with blood.

For proof of principle in a human system, the researchers decellularized human lung segments taken from a tissue bank and used a mixed population of human lung epithelial and endothelial cells to create a recellularized lung section.

The Yale results were published in Science.

"The key positive outcome from our study is in showing that these engineered lungs can be transplanted and are able to participate in oxygen and carbon dioxide exchange with the blood," said Thomas Petersen, lead author on the paper and a postdoctoral associate in the Department of Biomedical Engineering at Yale. "Our study demonstrates that it is technically feasible to grow a functional lung using the organ's decellularized matrix."

"Rather than growing a piece of tissue in the lab and just placing it somewhere in the body, the researchers have designed grafts that may be connected from day zero to the host circulatory system," said Soker. "This approach will primarily provide a viable alternative to organ donation and will also help us to study how bioengineered tissues respond to molecular signals from the host environment."

He noted that the main difference from earlier liver and lung tissue constructs is that the host would be able to send and receive signals from these engineered organ grafts through the circulatory system.

"The common theme in these two papers is that the researchers recognized that there are currently no good substitutes for the body itself as a bioreactor for evaluating graft function and that the 3D extracellular 


\section{Box 1. From hearts to lungs and beyond.}

While groups at Harvard Medical School and Yale University built their functional liver and lung grafts using only rat cells, another Harvard team has created a functional lung graft using a mixture of human and rat cells. ${ }^{5}$ Those researchers, who published their findings in Nature Medicine, used the decellularized matrix of a rat lung as a scaffold and seeded it with human umbilical vein endothelial cells and rat fetal lung cells.

The chimeric graft carried out oxygen and carbon dioxide exchange with blood for the duration of the six-hour experiment in rats. The researchers did not detect parenchymal bleeding, pulmonary edema or air leakage in the lung grafts in the first hour after transplant. However, evidence of pulmonary edema emerged at later time points.

Histological analysis of the lung grafts following the transplant experiment revealed no evidence of interstitial hematoma or thrombus formation. But the researchers did detect proteinaceous fluid accumulation in the alveolar spaces and distal airways - a sign of pulmonary edema.

By comparison, the Yale group's rat lung transplantation experiment described in Science was carried out for up to two hours, during which the researchers detected only modest bleeding into the airways of explanted grafts.

"Our work further explores the in vitro culture conditions, cell-seeding strategies and types of cells that we can use to create a functional lung graft," said Harald Ott, corresponding author on the Nature Medicine paper and an instructor in surgery at Harvard Medical School. "The key challenges ahead are to develop better cell-seeding strategies, obtain a better understanding of the biology of these decellularized matrices and find the types of cells to use to seed the matrix."

He added: "There are about 40 different cells in the lung, so we will need to figure out how to get various cells to where they're supposed to be."

Ott said the group's next steps are to scale up the current approach to create a human-sized graft using decellularized lung matrices and to evaluate lung grafts recellularized with adult human stem cells in small animal models.

In addition to the lung, he said his group is developing scale-up protocols to create human-sized hearts and pancreases.

Ott said the University of Minnesota holds an issued patent covering the decellularization and recellularization of organs and tissues. The patent has been licensed to Miromatrix Medical Inc., a regenerative medicine startup developing human replacement organs and biological tissue replacement products.

Harvard Medical School has filed multiple patent applications covering the bioreactors and culturing methods used to create the lung, heart and pancreas grafts. The patents are available for licensing through Partners HealthCare Research Ventures and Licensing.

matrix is the ideal structure to use for reconstructing functional tissues and organs," said Stephen Badylak, a professor in the Department of Surgery at the McGowan Institute for Regenerative Medicine at the University of Pittsburgh. "The biggest advantage of the decellularization approach described in both papers is that we don't have to re-engineer the 3D matrix for the organ."

Earlier this month, Harald Ott and colleagues published findings in Nature Medicine similar to those described by the Yale group (see Box 1, "From hearts to lungs and beyond"). Ott is an instructor in surgery at Harvard Medical School and lead author on the 2008 paper describing the beating rat heart.

\section{Optimizing the protocol}

Uygun said his group is now developing protocols to incorporate additional cell types into the engineered liver graft. Ongoing studies are focused on optimizing the team's cell-seeding protocol and developing strategies to cover graft vasculature with endothelial cells.

Massachusetts General Hospital has filed a patent covering the crea- tion of decellularized liver matrices and methods for obtaining cells from cadaveric livers. The work is available for licensing through Partners HealthCare Research Ventures and Licensing, the technology transfer arm for Massachusetts General Hospital and Brigham and Women's Hospital at Harvard Medical School.

Petersen's group is now refining its seeding and culturing protocols to improve blood vessel coverage in the lung graft. "With our current approach, we get good cell coverage across the vascular network within the graft, but this coverage is not perfect. Blood clots will form at any location on the matrix that isn't covered by cells," he told SciBX.

The group also hopes to increase the amount of functional surfactant being produced by the alveolar cells in the lung graft. Surfactants are important for efficient gas exchange.

Yale has filed a patent application covering the methodology described in Science. The work is also available for licensing.

Lou, K.-J. SciBX 3(28); doi:10.1038/scibx.2010.850

Published online July 22, 2010 


\section{REFERENCES}

1. Uygun, B.E. et al. Nat. Med; ; published online June 13, 2010; doi:10.1038/nm.2170

Contact: Korkut Uygun, Harvard Medical School, Boston, Mass. e-mail: kuygun@partners.org

2. Petersen, T.H. et al. Science; published online June 24, 2010; doi:10.1126/science.1189345 Contact: Laura E. Niklason, Yale University, New Haven, Conn. e-mail: laura.niklason@yale.edu

3. Badylak, S.F. Biomaterials 28, 3587-3593 (2007)

4. Ott, H.C. et al. Nat. Med. 14, 213-221 (2008)

5. Ott, H.C. et al. Nat. Med.; published online July 13, 2010; doi:10.1038/nm.2193
Contact: Harald Ott, Harvard Medical School, Boston, Mass. e-mail: hott@partners.org

COMPANIES AND INSTITUTIONS MENTIONED

Brigham and Women's Hospital, Boston, Mass.

Harvard Medical School, Boston, Mass.

Massachusetts General Hospital, Boston, Mass.

Miromatrix Medical Inc., Minneapolis, Minn.

Partners HealthCare, Boston, Mass.

University of Minnesota, Minneapolis, Minn.

University of Pittsburgh, Pittsburgh, Pa.

Wake Forest Institute for Regenerative Medicine,

Winston-Salem, N.C.

Yale University, New Haven, Conn. 